\title{
Philosophiques
}

\section{Pierre Hadot, Plotin : Traité 9 (VI 9), [Introduction, traduction, commentaire et notes], Paris, Cerf, 1994, 252 p.}

\section{Georges Leroux}

Volume 24, numéro 1, printemps 1997

Avez-vous lu Rawls?

URI : https://id.erudit.org/iderudit/027430ar

DOI : https://doi.org/10.7202/027430ar

Aller au sommaire du numéro

Éditeur(s)

Société de philosophie du Québec

ISSN

0316-2923 (imprimé)

1492-1391 (numérique)

Découvrir la revue

Citer ce compte rendu

Leroux, G. (1997). Compte rendu de [Pierre Hadot, Plotin : Traité 9 (VI 9),

[Introduction, traduction, commentaire et notes], Paris, Cerf, 1994, 252 p.]

Philosophiques, 24(1), 185-187. https://doi.org/10.7202/027430ar

Ce document est protégé par la loi sur le droit d'auteur. L'utilisation des services d'Érudit (y compris la reproduction) est assujettie à sa politique d'utilisation que vous pouvez consulter en ligne.

https://apropos.erudit.org/fr/usagers/politique-dutilisation/ 


\title{
COMPTES RENDUS
}

\author{
Pierre Hadot, Plotin: Traité 9 (VI 9), [Introduction, traduction, \\ commentaire et notes], Paris, Cerf, 1994, 252 p.
}

La publication du traité 9 , intitulé dans le catalogue porphyrien de la Vita Plotini " Du Bien ou de l'Un ", constitue la troisième contribution de Pierre Hadot à son édition commentée des Ennéades de Plotin. Amorcée en 1988 par la publication du traité 38 (VI 7), intitulé Comment la multiplicité des Idées a été produite et sur le Bien, cette série offre également une édition du traité 50 (III 5), Sur Eros, parue en 1990. Guand elle sera complétée, cette série comprendra l'ensemble des traités dans l'ordre chronologique. Ceux-ci sont présentés sans titre, par leur seul numéro dans l'énumération de Porphyre, ce qui représente une manière un peu abstraite d'aborder les textes, mais qui se veut plus fidèle au propos de Plotin. Plusieurs traitès sont en effet consacrés à des sujets diversifiés. Cette édition nouvelle veut répondre aux besoins des lecteurs des Ennéades, en leur foumissant les éléments à la fois philologiques et philosophiques nécessaires à l'interprétation du texte fondateur du néoplatonisme.

Il ne faut pas hésiter à qualifier l'entreprise de Pierre Hadot de grand projet d'édition, car les trois volumes qui sont à notre disposition apportent beaucoup plus qu'une traduction commentée. La structure de chacun est identique : on trouve d'abord une introduction élaborée, dans laquelle la portée philosophique du traité est discutée et rapportée non seulement à l'ensemble des Ennéades, mais également à la pensée tardo-antique. Les sources sont présentées de manière succincte, mais on les retrouve de manière plus élaborée dans le commentaire. Cette introduction permet de mesurer les grands enjeux de l'interprétation de chaque traité et sa contribution spécifique aux Enréades. Elle est suivie de notes sur le texte grec, d'un plan détaillé du traité et d'une traduction annotée. Présentée avec un soin exceptionnel, cette deuxième partie offre tous les éléments de discussion du texte grec nécessaires au lecteur désireux de rapporter la traduction à l'édition critique de P. Henry et H.-R. Schwyzer. Les notes portées au bas de la traduction exposent les choix du traducteur, en plus d'offrir les éléments habituels de lieux parallèles et de sources antiques. Le lecteur moins intéressé par les questions philologiques trouvera son profit surtout dans la troisième partie, dans laquelle Pierre Hadot propose un commentaire détaillé du texte sur le plan philosophique. La méthode employée ici repose pratiquement sur l'exégèse néoplatonicienne antique ellemème, dans la mesure où elle recourt souvent à la position d'un lemme pour développer l'interprétation. Celle-ci se révèle très sensible aux problèmes particuliers de la composition des Ennéades et elle n'épargne aucun effort pour restituer aux doctrines de chaque traité leur environnement spéculatif dans l'ensemble formé par les cinquante-quatre traités.

La présentation du traité 9 ne fait pas exception à cette structure établie dès la parution du traité 38. Dans son introduction. Pierre Hadot fait valoir la richesse et l'importance des thèmes relatifs à la pensée plotinienne de l'Un. Il s'agit d'un traité qui appartient à la première production de Plotin, avant 
l'arrivée de Porphyre dans l'école. Placé par Porphyre à la toute fin de l'ordre systématique (VI 9), ce traité fait suite dans cet ordre à deux grands traités de nature théologique, 38 (VI 7) et 39 (VI 8) dans lesquels Plotin montre à la fois la nécessité et les apories d'une pensée de l'Un. Mais là où les traités précédents ne font qu'esquisser les prolongements mystiques de la métaphysique et de la théologie rationnelle négative, le traité 9 (VI 9) engage résolument son lecteur sur le chemin de la contemplation et de l'union mystique. En le plaçant en dernier dans l'ordre de lecture prescrit pour les membres de l'école, Porphyre a sans doute voulu insister sur l'importance de concevoir toute la propédeutique philosophique des traités èthiques, physiques et métaphysiques comme un exercice devant conduire ultimement à un dépassement spirituel. Rappelons que la notion même de métaphysique s'entend ici de la spéculation sur les objets théologiques (l'Âme, l'Intellect et l'Un) et qu'en tant que tel, l'Un ne saurait constituer un objet.

Attentif à la structure rhétorique du traité. Pierre Hadot montre que l'exposé est divisé en deux parties, structure dans laquelle la deuxième reprend en la renforçant la première. Cette structure est celle d'une remontée spéculative des êtres vers l'Un, c'est-à-dire vers le principe de l'unité. La démonstration de la nécessité de poser une réalité transcendante n'est cependant pas détachable de l'expérience de l'Un : "...on ne peut saisir l'Un qu'en ètant soimême " un ", c'est ainsi que Pierre Hadot résume la proposition spéculative qui lie l'affirmation théologique et l'expérience mystique. Le traité 9 comporte donc une dimension anagogique fondamentale : il ne s'agit pas seulement d'élaborer la théologie négative, toujours déjà rationnelle dans son recours à la discursivité particulière de la négation et de l'aphérèse, mais d'indiquer à travers elle le chemin à parcourir pour parvenir à la coïncidence avec l'Un. Hadot insiste avec raison sur limportance du passage consacré à ce thème $(7,1-11,51)$, en disant qu'il s'agit de la pièce maitresse du traité. Le concept déterminant pour cette élaboration est celui de présence $(7,5)$, ou encore de coïncidence, conduisant à l'union (sunousia, 7, 24). Le thème correspondant de l'amour manifeste en quel sens l'Un est saisi comme Bien et rend possible une expression de l'union comme saisie et fusion.

Dans cette riche introduction, qui présente une synthèse magistrale des thèmes de la théologie négative et de l'expérience mystique, thèmes auxquels Pierre Hadot a consacré de nombreuses études antérieures, je voudrais m'arrêter à une question particulièrement difficile pour l'herméneutique des Ennéades. L'interprétation de la métaphysique de Plotin doit faire large la place de l'altérité et de l'absolue différence. Dans le cas de l'Un, tout langage tenu à son sujet pourrait n'être qu'un langage relatif à nous-mêmes. Hadot consacre à cette proposition $(3,49-54)$ une partie intéressante de son introduction et de son commentaire. L'affirmation de l'ineffabilité. qui deviendra si importante dans la theologie ultérieure, constitue en fait une thèse sur le langage. En écrivant que " tout ce qui est dit dans le discours théologique sur l'Un [...] n'est finalement qu'une description des ètats dans lesquels se trouve laame par rapport à l'Un ", et en affirmant que cette thèse nouvelle ne se retrouvera que beaucoup plus tard chez Damascius (Des Premiers Principes, 8, 17 WesterinkCombès), Hadot met en relief un des problèmes déjà relevés par $P$. $O$. Kristeller dans sa thèse de 1929 sur l'âme. Une radicalisation de cette proposition du traité, qui voudrait tenir compte de la faiblesse inhérente du discours humain, ne serait-elle pas que l'ensemble de la métaphysique (de la théologie au sens néoplatonicien) a d'abord un sens que Kristeller appelait " actuel ". c'est-à-dire relatif à l'expérience de l'âme, et seulement ensuite un sens métaphysique 
objectif, relatif à l'existence des réalités transcendantes ? L'immense intérêt du commentaire de Pierre Hadot est d'être partout attentif à la portée spirituelle, c'est-à-dire d'emblée * actuelle ", du texte de Plotin.

Son commentaire des passages mystiques du traité est en tous points admirable. La méthode de ce commentaire mériterait à elle seule une analyse détaillée : l'interprétation procède par segments limitès, dans lesquels Pierre Hadot met en place tous les éléments nécessaires pour relier une proposition particulière à toutes celles qu'elle croise dans l'argument et dans le texte d'ensemble. Cette méthode se fonde donc sur un système de renvois très élaboré, qui exige de son lecteur un effort considérable, mais la précision conquise de la sorte apporte sa récompense : le texte est lu, pour ainsi dire, de l'intêrieur et jamais l'interprète n'adopte à son égard une position de surplomb. Le commentaire devient pour le lecteur non seulement un véritable travail analytique, mais un cheminement rigoureux dans les tracés multiples de l'œuvre. Le rapport à l'érudition n'est jamais lourd ou encombrê et il est donc possible de lire ce commentaire, comme je l'ai dit plus haut, comme on lit un commentaire ancien, c'est-à-dire pour sa portée spirituelle et non seulement comme un travail savant.

La traduction proposée s'appuie sur les deux éditions de Henry et Schwyzer : l'editio major de 1973, l'editio minor de 1982. Pierre Hadot intervient sur le texte dans 45 passages: dans certains cas, il corrige des erreurs évidentes, mais dans plusieurs passages, il propose des variantes intéressantes (par exemple en 2,17 ou 8,38 ). Ce travail philologique permet de voir que l'editio minor de Oxford ne peut pas ètre lue de manière entièrement indêpendante de l'editio major. Les notes de Pierre Hadot renvoient souvent au travail de P. A. Meijer (Plotinus on the Good or the One (Enreads, V 9). An Analytical Commentary. Amsterdam, J. C. Gieben, 1992), étude pour laquelle il manifeste une grande admiration, mais je n'ai pu à ce jour la consulter personnellement. La traduction est, comme dans les traités précédents, d'une remarquable précision et il faut se réjouir du retour à la traduction de noûs par Intellect, au lieu de Esprit. Une bibliographie choisie ainsi que des index d'auteurs anciens et modernes, de termes grecs et de thèmes généraux complètent ce livre, qui constitue une contribution d'une exceptionnelle richesse à l'étude des Ennéades.

Georges Leroux

Département de philosophie Université du Québec à Montréal 